\title{
Téoros
}

Revue de recherche en tourisme

\section{Identité culturelle et stratégie de développement d'une image touristique de marque}

\author{
Un cas, la Vallée de la Matapédia
}

\section{Guy Massicotte et Marièle Cantin}

Volume 7, numéro 1, mars 1988

Cultures régionales et tourisme

URI : https://id.erudit.org/iderudit/1080425ar

DOI : https://doi.org/10.7202/1080425ar

Aller au sommaire du numéro

Éditeur(s)

Université du Québec à Montréal

ISSN

0712-8657 (imprimé)

1923-2705 (numérique)

Découvrir la revue

Citer cet article

Massicotte, G. \& Cantin, M. (1988). Identité culturelle et stratégie de

développement d'une image touristique de marque : un cas, la Vallée de la

Matapédia. Téoros, 7(1), 11-13. https://doi.org/10.7202/1080425ar d'utilisation que vous pouvez consulter en ligne. 
Un cas, la Vallée de la Matapédia

Guy Massicotte, avec la coll. de Marièle Cantin*

\section{Identité culturelle et stratégie de développement d'une image touristique de marque}

\section{Le Projet Initiative \& Développement}

rapports entre développement régional et développement touristique, celui-ci devant favoriser celui-là, en s'appuyant sur l'identité culturelle des entites régionales et en favorisant leur epanouissement ${ }^{(1)}$. Cette experience, nous l'avons faite, pour ainsi dire comme à rebours, en partant d'une démarche de développement régional qui nous a conduit entre autres choses à une démarche de développement touristique.

Notre intervention s inscrit dans une double perspective: d'une part, l'implication de l'Université du Québec à̀ Rimouski dans le milieu régional, et singulièrement les activités de la Maitrise en développement régional, du Groupe de recherche interdisciplinaire en développement de l'Est du Québec (GRIDEQ) et du Projet Initiative \& Développement (ID) - sous l'égide duquel a été réalisée cette intervention - et d'autre part, la volonté du milieu matapédien de structurer ses orientations et actions de développement dans un cadre plus precis et mieux défini. lisme et au court terme; citées;
M. Mrssicotte et Mme Cantin font partie du Groupe de recherche interdisciplinaire an developpement de l'Est du Quebec, Universite du Quebec a Rimouski.
Les concepts d'intervention qui sont à la base du projet Initiative \& Développement ont été élaborés suite à deux constats: 1) la difficulté des universitaires de s'arrimer aux besoins et aux priorités des milieux locaux et régionaux; 2) la difficulté de ces derniers d'assumer les travaux de réflexion et de recherche nécessaires à la prise en charge de leur développement. Ils s'énoncent ainsi:

- l'analyse et la prospective d'ensemble afin de faire échec aux tendances par trop naturelles à la sectorialisation, au loca-

- la planification, la recherche et le développement comme support au développement régional de façon à identifier et à supporter des projets dont la pertinence est démontrée et dont la faisabilité et les conditions de réalisation sont bien expli-

- la continuité et les interactions permanentes avec les intervenants du milieu, de façon à réagir contre les interventions trop spécialisées et trop lointaines de certaines firmes de consultants, et celles, souvent trop théoriques et trop générales de Ia recherche universitaire proprement dite: chaque activité doit se dérouler dans une perspective de continuité avec des intervenants identifiés qui deviennent des partenaires à part entière de l'intervention;

- l'utilisation maximale des ressources humaines et physiques du milieu en vue de leur mise en valeur, laquelle constitue la finalité ultime du Projet Initiative \& Développement.

C'est à la lumière de ces principes que nous nous sommes engagés dans le processus d'élaboration d'un Plan de développement régional pour la Vallée de lạ Matapédia.

\section{L'Ader de la Matapédia}

Rappelons que la Vallée de la Matapedia, dont l'espace correspond approximativement à celui de la MRC du mểme nom, s'étend de la tête du Lac Matapédia jusqu'à l'embouchure de la Ristigouche, a la naissance de la Baie des Chaleurs. Elle s'étend done de part et d'autre du réseau hydrographique constitué par le Lac et la Rivière Matapédia, cet axe étant également celui de la route 132 et du Chemin de fer du Canadien national. Les principales municipalités sont localisćes sur cet axe, où l'on retrouve, du Nord au Sud, Sayabec, Val Brillant, Amqui, Causapscal et Lac-au-Saumon. De part et d'autre de la vallée, les Appalaches se déploient en plateaux et parfois en massits assez imposants, comme celui de Val d'Irène, sur lesquels se sont ćtablis plusieurs paroisses et villages agroforestiers.

Colonisée depuis le dernier tiers du XIXe siècle suivant un patron s'approchant de la quintessence de ce que les sciences humaines appellent le "système agro-forestier", la région est encore aujourd'hui largement tributaire des activités agricoles et forestierres. Depuis le milieu du siècle, la Vallée a perdu le tiers de sa population, celle-ci passant de 34000 habitants en 195 I a 22000 en 1986. Il s'agit du principal problème de la région: comment stabiliser la population en fournissant des emplois intéressants aux citoyens, notamment aux jeunes?

Depuis plusicurs décennies, les leaders de la région sont activement engagés dans des actions revendicatrices et développementales. Les Opérations Dignité, nées sur les hauteurs des pays de Matane et de Rimouski ont fortement influençé les Matapédiens qui mettaient sur pied dans les années 1970 la première Société d'exploitation des ressouroes qui demeure encore des plus dynamiques, et qui compte déjả à son actif des réalisations importantes dans le domaine de l'aménagement forestier.

Si le secteur des services, et notamment celui des services publics s'est relativement bien développé, et si l'économie agricole ct forestière a plutôt bien réussi son intégration au processus de modernisation qui a caractérisé ces activités dans l'ensemble du Québec, le secteur industriel demeure plus faible. C"est done vers un renforcement du secteur secon= daire et une diversification de l'exploitation des ressources naturelles que se sont orientés les efforts de développement du milieu. Dans cette perspective, le tourisme, qui figure depuis longtemps à l'inventaire des potentiels de développement du milieu, suscite bien entendu beaucoup d'intérêt: on le considère sous l'angle d'une ressource primaire à exploiter ou celui d'un élargissement du secondaire vers le tertiaire moteur.

Parmi les organismes et institutions qui exercent une influence au sein du milieu matapédien, l'Ader de la Matapédia occupe une position stratégique. Crée en vertu du programme des sociétés CLE du gouvernement fédéral, cette Agence apporte un support 
financier et technique aux entreprises de la région. Ses dirigeants étaient bien à même de constater les problèmes crées par l'absence d'orientations et de stratégies de développement pour l'ensemble de la région.

\section{Plan de développement régional et tourisme}

Une demande fut donc adressece à l'Université du Québec à Rimouski pour une contribution a l'elaboration d'un Plan de développement. Conformément aux principes de base du Projet ID, on se refusa à une approche de consultants, pour préconiser plutôt: 1) une prise en charge par le milieu lui-même de son Plan de développement; 2) le support professionnel de l'Université à un groupe de travail mandaté pour préparer un Plan, support prenant la forme d'un appui scientifique et logistique d'un chercheur en développement régional et d'une étudiante à la Maîtrise en développement régional.

La préparation du Plan de développement fut pris en charge par un groupe de travail issu du milieu, les deux auteurs assurant le travail de recherche de données et de rédaction des textes. Bien entendu, le Plan vise l'ensemble des secteurs de l'activité économique de la Vallée $e^{(2)}$. Mentionnons quelques caractéristiques significatives pour les activités de développement touristique:

- toute la logique du Plan est basée sur la connaissance et la comprchension des acquis, leur exploitation et leur prolongement dans des projets et des activités de développement;

- les acquis reconnus des Matapédiens concernent très largement les ressources naturelles agro-forestières, fauniques et touristiques: développer signifie donc, en l'occurrence, micux exploiter ces ressources:

- en matière touristique, le Plan ne va pas très loin, compte tenu des difficultés particulières qui affectent ce domaine et qu'on résumera plus loin, mais sur la base d'un double constat, éclatement des centres d'initiative et absence d'image de marque, il recommande le regroupement des intervenants en fonction d'une préoccupation de promotion du produit touristique régional.

Lors de l'Assemblée générale annuelle de l'Ader au mois de mai 1986 , la recommandation sur le tourisme reçut une haute priorite. Elle comportait deux objectifs: 1) susciter et soutenir les efforts de concertation; 2) développer et promouvoir une image régionale de marque. Objectifs qu'elle proposait d'atteindre en constituant un groupe de travail voué à la promotion de la région et par le support à des projets spécifiques de développement touristique ayant comme préoccupation la mise en valeur de traits régionaux originaux.

Comme suite au Plan de développement de l'Ader, il avait été décidé, pour chacune des priorités identifiées, qu"un groupe de travail serait constitué pour la mise en oeuvre des objectifs. C'est ainsi qu'un comité regroupant des intervenants touristiques du milieu, ainsi que les deux auteurs, fut mis sur pied. Les travaux de ce comité, commencés depuis quelques mois, se veulent une réponse aux problèmes de concertation et de développement par l'élaboration et la mise de l'avant d'une image de marque correspondant aux traits spécifiques du milieu matapédien.

\section{Multiplicité des centres d'initiative et absence d'image de marque}

Parallèlement aux travaux du Plan de développement de l'Ader, la MRC de la Matapedia entreprenait la confection de son Schéma d'aménagement. Dans ce contexte, une étude fut réalisée dans le cadre du Projet Initiative \& Developpement sur les potentiels physiques, économiques, sociaux, culturels et politiques du développement touristique $^{(3)}$. Parmi les principales conclusions de l'étude, on notait:

- l'existence d'un riche potentiel, exprimé notamment en termes de quantité de projets réalisés ou suggérés;

- l'absence de concertation entre des intervenants nombreux, diversifiés et répartis sur l'ensemble du territoire de la MRC.

- l'absence d'image de marque susceptible d'orienter et de stimuler les efforts de promotion et de développement.

L'étude concluait aussi en la nécessité de mandater un organisme régional pour assurer le leadership du développement touristique.

Au début de l'automne 1986, on se retrouvait devant la situation suivante:

- un ensemble d'intervenants de premier niveau, hôteliers, restaurateurs, corporations locales, avec des objectifs sectoriels ou municipaux, essayant chacun de développer ses projets en fonction de sa vision particulière. Un de ces intervenants, Tour Mat, ayant eu dans le passé une volonté réelle de concertation, de planification et de développement régional, mais n'étant plus en mesure de l'assumer;

- un Plan de développement touristique pour la Gaspésie, réalisé par la firme Roche, plan plus ou moins identifié à l'Association touristique régionale, et dans lequel les Matapédiens ne semblaient pas se retrouver;

- la MRC consciente de la nécessité, ne serait-ce que pour des fins d'aménagement, mais encore davantage dans une perspective de développement, d'une meilleure concertation et d'une vision plus claire des orientations à privilegier:

- l'Ader, dans le cadre de son Plan de développement, est parvenu à la même conclusion;
- la mise sur pied, au méme moment, d'une Corporation de développement économique (COPEM), à vocation régionale et intéresséc à jouer un rồle moteur au plan du développement touristique;

- les deux niveaux de gouvernement continuant d"insister pour une meilleure concertation et mettant de l'avant de nouvelles approches incitant le milieu à identifier et a promouvoir ses propres priorités.

Il y eut pendant quelques mois beaucoup de consultations informelles, quelques réunions formelles, dont l'une assez tumultueuse. Dans ce débat, les chercheurs impliqués ont découragé toute vellếité de créer un nouvel organisme. Il paraissait en effet préférable qu'un organisme existant prenne le leadership en s'imposant par la crédibilité de sa démarche. Il faut dire que les organismes intéresscés au développement, MRC, ADER. COPEM, ont fait preuve de beaucoup de maturité, et tout en demeurant étroitement reliés â la démarche, se sont facilement talliés au leadership de la COPEM. Un groupe de travail COPEM, ADER, MRC, ID a été mise en place et un consensus tend à prendre forme quant à sa stratégie d'intervention:

- impliquer les intervenants de premier niveau par l'information, la consultation, l'interaction ouverte, plutồt que par leur participation directe, étant donné qu'ils sont de par la nature même de leurs activitếs en conflit d'intérêt et en situation de concurrence les uns avec les autres;

- oeuvrer dans une perspective de comité d'action, plutốt que dans celle d'un Comité de coordination, et s'imposer par la crédibilité et l'efficacité plutôt que par des reconnaissances formelles;

- exercer une action de concertation et de développement par la mise de l'avant d'une image touristique de marque, cette absence d'image de marque étant identifiese comme le handicap majeur aux efforts de concertation, de promotion et de développement.

\section{Identité culturelle et développement}

Au niveau du projet ID lui-même, touțe une réflexion est amorcée depuis quelques annóes sur les rapports entre identité culturelle et développement. Il apparaît, en effet, que pour résister aux effets négatifs des forces exogènes qui les affectent, les milieux locaux et régionaux doivent prendre conscience de leur réalité propre et s'engager dans des actions qui tiennent compte des forces et des faiblesses, des potentiels et des contraintes du milieu qu'ils habitent et qui les habite. Entre l'individu et l'humanité dans son ensemble, il existe toute une hierarchie d'appartenance qui operrent chacune à des degrés d'efficacité variable. L'un des paramètres de cette efficacité est précisément la conscience d'une capacité d'être et de devenir dont le contenu est fait de la connaissance 
de soi et de ses aspirations réelles - et réalistes.

Soutenir le développement régional, au niveau d'une communaute locale et microrégionale, implique donc que l'on en connaisse les caractères propres et les forces endogènes qui faworisent ou contraignent son auto-développement. Cette prise de conscience, en elle-même libératrice d'énergie, est préalable à tout effort de planification réaliste. Faut-il ajouter que cette prise de conscience doit englober les différentes composantes de la vie collective et la manière dont elles interagissent entre elles, et notamment sur la culture, qui se constitue des perceptions, des savoirs, des savoir-faire, des croyances, des valeurs, des idéologies, des individus, des groupes, et de la communauté elle-même dans să spécificité spatiale et institutionnelle? Pour répondre à cette exigence, un projet de recherche dont le sujet est "Espace, culture et développement" a ćté entrepris. Il est non seulement inévitable mais souhaitable que ces préoccupations influencent une intervention spécifique comme celle du développement touristique.

\section{Développement touristique et image de marque}

A la lumière de ces considérations, le Comité d'action $s^{\top}$ est engagé dans un travail de réflexion visant la définition de cette image de marque. Au point de départ, il fut convenu qu'elle devait comporter les caractéristiques suivantes:

- bien exprimer le potentiel du milieu naturel et humain de la région;

- représenter une source d'attraction réelle pour un créneau identifié du marché;

- recevoir l'adhésion des intervenants politiques, économiques et sociaux du milieu;

- être capable d'engendrer un plan d'action de développement tant au niveau du produit touristique lui-même (offre) que de sa mise en marché.

Puisque nous cherchons à développer le tourisme dans la Matapédia, il s'agit de repérer les attraits, les ressources susceptibles de presenter un intérêt pour des personnes de l'extérieur. Ces attraits, tout en étant profondément identifiés au milieu, doivent lui être relativement spécifiques de manière à servir de point d'appui à la mise en valeur d'un produit touristique original.

Il apparaît que le milicu naturel (rivières, montagnes, lacs, forets...) et que le milieu culturel (économie agro-forestière) constituent deux caractéristiques essentielles du milieu matapedien sur lesquelles il faut compter.

Au plan du milieu naturel, il existe déjà des acquis importants en ce qui a trait aux activités de sport et de loisirs (ski, pêche au saumon, ...) alors que le milieu socio-culturel se caractérise par des valeurs d'attachement et de fierté par rapport à des savoir-faire et des modes de vie qui constituent une richesse à exploiter face à la modernité et au déracinement.

En conséquence, il fut convenu de mettre de l'avant, tout au moins à titre d'hypothèses de travail, les deux orientations suivantes:

- les activités de sport et de loisir en milieu naturel;

- la découverte des savoir-faire et des genres de vie propres à l'économie et à la société agro-forestière.

Ces deux orientations sont susceptibles de rejoindre des créneaux importants du marché. Dans le premier cas, il existe dans le Bas St-Laurent et la Gaspésie, dans les Maritimes, ainsi que dans les bassins urbanisćs du Québec, des adeptes des sports et loisirs de plein air susceptibles d'être attirés par les perspectives d'une palette d'activités diversifiées, bien organisées, non encombrées, à prix raisonnable et répondant aux besoins de la famille.

Dans le second cas, il existe un très grand nombre de Québécois maintenant urbanisés, dont les parents et les grands-parents sont issus de l'économie et de la société agroforestiere. Ces gens sont fäcilement intéressés par la perspective de retrouvailles, surtout si l'on peut y associer loisir et détente.

En regard de la mise en marché, ces deux orientations ne sont pas exclusives l'une de l'autre, mais se renforcent au contraire l'une et l'autre, chacune constituant un attrait sccondaire pour ceux et celles surtout interessés-es par l'autre.

Ces orientations correspondent à des valeurs, à des idées et à des infrastructures déjă existantes dans le milicu. Elles peuvent susciter l'adhésion des décideurs locaux sans changement majeur des mentalites. Il faudra d'ailleurs plutôt les convaincre que des choses aussi simples peuvent avoir leur pesant d'efficacité. Il ne faudra cependant rien négliger pour diffuser dans le milieu un discours clair, simple et cohérent afin de créer un mouvement d'adhésion à ces orientations. Elles peuvent enfin servir à structurer un plan d'action dans le prolongement de oe qui existe déjă.

Ces hypothèses d'image de marque devront servir à élaborer un programme d'action que le comité devrait mettre en oeuvre dans les prochains mois. A cet égard, il faudra agir sur deux plans:

\section{au plan du produit touristique:}

- inventorier les attraits, services, ressources disponibles en fonction de chaque orientation:

- identifier les carences autant dans ce qui existe déjà, que dans ce qui pourrait ètre ajouté;

- proposer aux intervenants des correctifs á apporter et/ou des projets de dévelop- pement à implanter, et suivre l'évolution de leur mise en oeuvre.

au plan du marketing:

- élaborer des outils de promotion en fonction de marchés-cibles;

- élaborer et mettre en oeuvre des actions promotionnelies pertinentes.

\section{Un travail long et difficile}

Le développement touristique dans la Vallée de la Matapédia ne peut se concevoir et se mettre en oeuvre sans s'insérer dans une perspective plus large qui met en cause l'ensemble des processus de développement. Or, ceux-ci se caractérisent par la nécessaire utilisation des potentiels et des acquis régionaux dans le cadre d'actions de développement faisant appel aux forces vives de la région. Ces actions exigent une connaissance eclairée du milieu, ainsi que des orientations à privilegier et à mettre en oeuvre. C"est dans. cette perspective que nous intervenons dans la Vallée de la Matapédia, avec l'espoir que les démarches engagées se poursuivent dans un esprit de continuité, sans lequel toute action n'est qu'agitation.

Au terme d'un long processus, les leaders de la Vallée de la Matapédia disposent d'un cadre et d'un plan d'action, d'une orientation qui correspond bien à la réalité du milieu, et qui apparait assez riche pour engendrer des améliorations au produit luimême et à sa mise en marché. Il faut souhaiter que l'action entreprise se poursuive. $f$

\section{Notes explicatives:}

(1) BELANGEA, Marcel, "Une question neuve er dif ficlie. La regianalisation touristique au Quebec". Téoros, V. 1, 1986, pp. $2-4$.

12) ADER DE LA MATAPÉDIA, Plan de développetment socio- Economique de la Vallée de la Matapédia. Amquii: mai 1986,70 p. Le processus d'Alaboration du Plan a donné lieu à une thesse de maitrise en divasloppement rógional: CANTIN, Mariêle, Le plan de développement socio-économique de la Vallée de la Matapedia: vers un néo-pragmatisme dans Vapproche du développement?. Rimouski, UQAR, mai 1987, vii-163 p.

(3) ROY, Louises, Schéma d'aménagement. Secteur tourisme. MRC de la Matapédia. Rapport final, Aimouski, UOAR-ID, avril 1986, 71 p. 